\title{
Comportamento e sexualidade em estudantes universitárias de Salvador
}

Clésia Andrade Sadigursky*

Maria Hermelina Pita Souza**

Bruno Gil de Carvalho Lima***

\section{Resumo}

Introdução: A adolescência é a fase em que normalmente ocorre o início da atividade sexual, nem sempre sendo lembrados aspectos de planejamento e prevenção relacionados ao sexo. Objetivo: Estudar fatores ligados à sexualidade de universitárias de Salvador. Metodologia: Um estudo de corte transversal foi realizado através da revisão de 323 prontuários de estudantes da Universidade Federal da Bahia, entre 17 e 20 anos, atendidas no Serviço Médico Universitário Rubens Brasil, de janeiro de 1995 a dezembro de 1999. Estudaram-se a idade do início da atividade sexual, o uso de métodos contraceptivos, a ocorrência de gravidez, aborto e parto. Resultados: A iniciação sexual da maioria das estudantes ocorreu logo após a entrada na Universidade. $\mathrm{O}$ uso inadequado dos métodos protetores para

\footnotetext{
* Médica Pediatra/Hebiatra. Coordenadora dos Cursos de Especialização em Hebiatria da Faculdade de Medicina da UFBA e da Faculdade de Educação Olga Mettig.

** Médica Gincologista. Especialista em Hebiatria pel Faculdade de Medicina da UFBA.

*** Médico residente do setor de ginecologia da UFBA. e-mail: clesia@svn.com.br Recebido em 21.05.01 Aprovado em 08.06.01
} 
evitar as DST e a gravidez foi observado. O preservativo, método de escolha de $48 \%$ das clientes, provavelmente não foi utilizado de forma adequada nem em todas as relações sexuais. Constatou-se uma prevalência de $15 \%$ de gravidez, 33,3\% das quais abortadas. Discussão: Existe a necessidade da implementação de ações relacionadas à educação para a sexualidade para estudantes universitários, estimulando a compreensão da atividade sexual para o prazer e o adiamento da gravidez, para quando for possível planejá-la. Palavras-Chave: adolescência, sexualidade, contracepção.

\section{Abstract}

Introduction: The onset of sexual activity usually takes place during adolescence, but aspects of family planning and diseases prevention are seldom considered. Objective: Study factors related to university students ${ }^{\prime}$ sexuality in Salvador. Methodology: A cross-sectional study was carried on by means of the review of clinical files of 323 students of the Federal University of Bahia, aged between 17 and 20 years, that had used the University Medical Service Rubens Brasil from January, 1995 to December, 1999. Data regarding age of onset of sexual activity, use of contraceptive methods, and occurrence of pregnancy, abortion or delivery were studied. Results: The onset of sexual activity happened, for most of the students studied, right after admission to the university. Inadequate use of protection methods to avoid STDs and pregnancy was observed. Condom was the selected method for $48 \%$ of the clients, but probably not used adequately, nor in every sexual intercourse. There was a prevalence of $15 \%$ of pregnancy, 33,3\% of which terminated. Discussion: There is a necessity to develop actions related to education about sexuality to university students, stimulating the comprehension of sexual activity towards pleasure and postponing pregnancy to when it is possible to plan it.

Key-words: adolescence, sexuality, contraception.

\section{1 - Introdução}

A adolescência é uma época de novas expectativas e novos relacionamentos, marcada por crescimento rápido, desenvolvimento físico e psicossocial, maturação sexual, aquisição da capacidade reprodutiva e busca da identidade social e sexual, da definição do papel social determinado pelo ambiente sócio-cultural $(5,8)$. A Organização Mundial de Saúde (OMS) considera o período da adolescência compreendido na faixa etária entre 10 e 20 anos de idade.

As características da sexualidade na adolescência, entre outras questões, compreendem a falta de informações sobre sexualidade, o 
desenvolvimento de sentimentos de culpa, medos, imitação dos papeis sexuais dos adultos, preocupações com o próprio corpo, masturbação, intimidade no namoro e pouco equilíbrio emocional para ligações afetivas duradouras.

$\mathrm{Na}$ adolescência estrutura-se a auto-imagem, o perceber-se belo. Também a auto-estima: o sentimento de competência, de ser capaz de corresponder às expectativas da família e da sociedade. Transformam-se as emoções, com a necessidade de autonomia e de afastamento emocional dos pais. $\mathrm{O}$ pensamento e a cognição amadurecem, passa-se a ter a capacidade de raciocinar hipoteticamente, o que não era possível na infância (5).

Na puberdade, o corpo muda, a libido aumenta devido, inclusive, às altas taxas de hormônios sexuais que ativam os centros cerebrais $(2,4)$. A maioria dos homens e mulheres se iniciam sexualmente na adolescência (12). Freqüentemente, esses relacionamentos são temporários, o que os expõe aos riscos de gravidez inoportuna e de contaminação por doenças sexualmente transmissíveis (DST) $(22,23,24)$.

A sexualidade humana desenvolve-se do auto-erotismo até a capacidade de formação de vínculos de intimidade e compromisso com o outro, buscando a necessidade mais básica do ser humano: amar e ser amado $(5,8,13)$. As causas que condicionam a atividade sexual na adolescência podem estar representadas pela expectativa de novas experiências, aceitação pelo grupo, desafio à sociedade ou aos pais, recompensa ou punição, fuga da solidão, escape de outras pressões e busca do prazer físico.

As modificações de costumes sociais observadas a partir da década de 60 , representadas pelas aquisições sociais da mulher e a maior liberalidade sexual, são outros fatores que contribuem para o comportamento sexual na adolescência, assim como o advento dos métodos contraceptivos, a influência erotizante da mídia, a tendência para o casamento mais tardio, o prolongamento da vida escolar além do amadurecimento sexual precoce e falta de controle social $(22,23,24)$.

O relacionamento sexual premarital, atualmente vivenciado no ambiente sócio-cultural brasileiro, teve uma aceitação progressiva a partir dos anos 60 , com a derrubada dos valores sociais vigentes. Os meios de comunicação têm contribuído de maneira intensa para a sexualização da adolescência (18), e a iniciação sexual nos anos 90 ocorreu mais precocemente do que em décadas anteriores.

A sexualidade na adolescência, portanto, tem sido alvo de preocupações da saúde pública relacionada à atividade sexual precoce, à gravidez inoportuna e à dificuldade para o uso adequado dos métodos contraceptivos por esta população. O planejamento da gravidez está relacionado a condições sócio-econômicas (como classe social e nível de escolaridade) (8).

Estudos americanos demonstraram que $98 \%$ das mulheres solteiras aos 20 anos, que concluíram o $2^{Q}$ grau, usavam regularmente métodos contraceptivos e $82 \%$ planejaram a gravidez. Nos Estados Unidos ocorrem anualmente, cerca de 12 milhões de novos casos de DST. 75\% deste total ocorre em jovens com menos de 25 anos (8). 
Diversos estudos realizados na América Latina detectaram percentagens de atividade sexual entre 49 e $71 \%$ dos adolescentes, com predominância do sexo masculino (cerca de $80 \%$ ) em relação ao feminino ( 28 a $51 \%$ ). A idade da coitarca se concentrou entre 14 e 16 anos $(1,3,5,7,12,15,16,17,20)$. Embora as taxas de utilização de preservativos chegassem, em alguns casos, a $81 \%$, o uso regular e adequado de métodos contraceptivos foi, em outros, tão baixo quanto $5 \%$, com média de $36 \%$. Abortamentos foram referidos por aproximadamente $14 \%$ das jovens, estando uma maior prevalência associada a idade menor que 24 anos, ausência de companheiro e de religião $(9,21)$.

No Brasil, como em outros países, as adolescentes de classe média, principalmente, estudando para ter uma carreira profissional satisfatória, já cursando a Universidade, terão um longo período de dependência da família. Nessa fase, após a iniciação sexual, terão que abster-se de atividade sexual, ou ter a consciente certeza do exercício da sexualidade, sem finalidade reprodutiva, mas para o prazer sexual.

Os conceitos sobre a sexualidade que são ensinados referem-se ao perigo de contrair as doenças sexualmente transmissíveis ou da ocorrência da gravidez inesperada e inoportuna. Portanto, não lhes faltam conhecimentos sobre estes assuntos, mas falta-lhes atitude de proteção consigo mesmas, relacionado ao comportamento direcionado para a busca do prazer sexual consciente, deixando a reprodução para quando for possível planejá-la. Tem sido observada a necessidade de estimular a intimidade, a comunicação e a capacidade de negociar o momento sexual e o uso de protetores tanto para as DST, quanto para a prevenção da gravidez (18).

As garotas chegam às Universidade brasileiras entre os 17 e 18 anos de idade, portanto ainda no período da adolescência. Estudos sobre o comportamento sexual desta população são necessários para que sejam conhecidas as demandas causadoras de risco à saúde, ligadas ao exercício da sexualidade. O objetivo do presente trabalho foi descrever variáveis relacionadas à sexualidade de estudantes universitárias adolescentes de Salvador, Bahia, evidenciando possíveis aspectos que possam nortear iniciativas de orientação e educação para a sexualidade entre jovens.

\section{2-Metodologia}

Foi realizado um estudo epidemiológico retrospectivo, observacional e de corte transversal, de caráter descritivo, através da revisão dos prontuários médicos de 323 estudantes da Universidade Federal da Bahia, atendidas no Serviço Médico Universitário Rubens Brasil (SMURB) no período de janeiro de 1995 a dezembro de 1999.

Foram critérios de inclusão a idade entre 17 e 20 anos e o sexo feminino. Foram coletadas informações a respeito de atividade sexual, idade da 
iniciação sexual, uso de contraceptivos, gravidez e aborto. Devido à ausência de Comissão de Bioética ou congênere no SMURB, foi solicitada permissão à direção do órgão para a realização do trabalho, salvaguardando-se o sigilo dos dados dos prontuários, uma vez que não se identificaram as fichas de coleta.

Os dados foram analisados manualmente mediante a totalização das informações das fichas de coleta. Não foram utilizados métodos estatísticos de significância, dado o desenho descritivo do estudo, o qual não objetivava testar hipóteses.

\section{3 - Resultados}

Constatou-se que $65 \%$ das estudantes tinham vida sexual ativa, com idade da iniciação sexual concentrada entre 17 e 20 anos, conforme a tabela 1 .

Entre as estudantes com vida sexual ativa, $82 \%$ utilizaram algum método contraceptivo, tendo $58,7 \%$ referido o uso de preservativo, $33,2 \%$ uso de anticoncepcionais orais, $5,8 \%$ usavam anticoncepcionais injetáveis e 2,3\% seguiam o método de Ogino-Knaus (tabela 2).

A gravidez apresentou índices elevados, tendo ocorrido em $15,7 \%$ das adolescentes, sendo que 33,3\% referiram terem abortado (abortamento provocado), $9,1 \%$ não retornaram à consulta e $57,6 \%$ evoluíram para o parto.

\section{4-Discussão}

O presente estudo demonstrou que a iniciação sexual das adolescentes na Universidade Federal da Bahia, foi menos precoce do que tem sido observado na população geral. Entre os 17 e 18 anos de idade, observaram-se as maiores taxas de iniciação sexual, correspondente a $44 \%$, portanto coincidindo com o recente ingresso na Universidade.

Os resultados aqui apresentados demonstram que as adolescentes universitárias tenderam a iniciar a atividade sexual após o ingresso na Universidade, quando poder-se-ia esperar que dispusessem de um certo cabedal de informações acerca de gestação inoportuna, DST e métodos contraceptivos. De fato, a frequiência de uso de anticoncepcionais detectada foi maior do que na população geral, e o método preferido, o preservativo, tem a vantagem de também constituir uma barreira à transmissão das DSTs, ponto de importância tanto mais considerável quanto maior a labilidade dos relacionamentos de caráter emotivo-sexual na faixa etária em questão. Entretanto, muitas descuidaram-se, deixando de prevenir-se com o uso de métodos de proteção contra as DST/AIDS e contra a gravidez, muitas vezes não planejada, o que foi evidenciado pelo alto índice de gravidezes e 
abortos. A prevalência de uso de contraceptivos e a de gravidez foram conflitantes, o que provavelmente indica uso esporádico ou incorreto dos métodos escolhidos.

Ressalte-se, ainda, que embora quase $60 \%$ das gestantes tenham levado a gravidez a termo, isso não significa que se tratasse de gestações desejadas ou planejadas. As mudanças provocadas por um filho sem dúvida trazem consequiências danosas para os estudos universitários, e na idade da maioria das estudantes incluídas na investigação é provável a dependência econômica em relação aos pais, fatores que tenderiam a estimular o adiamento de uma gravidez.

Os resultados deste estudo, embora limitados pelo reduzido tamanho amostral, que pode ter contribuído para uma menor representatividade em relação à população universitária como um todo, demonstram a necessidade da implementação de ações a serem desenvolvidas para a prevenção dos agravos à saúde entre as estudantes universitárias, relacionadas à prevenção das DST/AIDS e da gravidez não planejada e conseqüente aborto não espontâneo. É preciso, ainda, garantir que tais ações sejam voltadas para os grupos de menor poder aquisitivo da comunidade universitária, visto serem os maiores usuários do serviço médico gratuito do SMURB, portanto melhor representados nos resultados do presente estudo.

O conceito de saúde e a maneira de abordar os clientes têm-se modificado nos últimos anos. Os profissionais de saúde começam a perceber a necessidade de substituir os modelos apenas biológicos e funcionais por atendimentos sistêmicos que incluem o contexto sócio-ambiental da doença e do doente, ou seja, o equilíbrio entre o indivíduo e os grupos sociais (11).

As condições de moradia, alimentação, higiene, imunizações, poluição, estresse, nível de informação, escolaridade, tensão emocional, autoestima, capacidade de tomar decisões e a qualidade dos relacionamentos afetivos são considerados, atualmente, variáveis que interferem na saúde individual e da coletividade $(2,4)$. A OMS define a saúde como "um estado de completo bem estar físico, mental e social, e não só como a ausência de enfermidades ou doenças". A finalidade desta definição é direcionar a compreensão das transformações nos estilos de vida pessoal e social, pontuando a capacidade dos indivíduos de buscar a saúde, visando a integridade biopsicossocial para a possibilidade de uma vida produtiva e harmoniosa (11).

Portanto, deve constituir uma prioridade educacional nos tempos atuais a elaboração de um programa de educação para a sexualidade, visando a promoção da saúde, a estruturação da auto-estima relacionada à sexualidade, à necessidade de estimular a mudança de atitude, privilegiando o diálogo e a negociação dos mecanismos de proteção contra os agravos à saúde entre os pares, relacionados à prevenção da gravidez inoportuna e do aborto nesta população. 


\section{REFERÊNCIAS BIBLIOGRÁFICAS}

1. ACOSTA RICO, S.L.; BERNAL, R. Actividad Sexual y Métodos Anticoncepcionais Utilizados por los Estudiantes de la Universidad Nacional de Colombia. Bogotá. S. 1; s. n. 1998. XI, 78 p.

2. BALEEIRO, M.C.; SIQUEIRA, M.S.; CAVALCANTI, R.C.; SOUSA ,V. Sexualidade do Adolescente: Fundamentos para uma Ação Educativa. Salvador: Fundação Odebrecht, 1999.

3. BENTO, I. Sexualidade e DST/AIDS em uma População Universitária. J. Bras. Doenças Sex. Transm. 1999; 11(2):17-25.

4. CANESSA, P. Manual para la Educación en Salud Integral del AdolescenteOrganización Panamericana de la Salud, Organización Mundial de la Salud Programa de Salud de la Familia Y Población, 1997.

5. CHIPKEVICH, E. Puberdade e Adolescência: Aspectos Biológicos, Clínicos, e Psicossociais. São Paulo: Roca, 1994.

6. EDGARDH, K. Sexual Behavior and Early Coitarche in a National Sample of 17-Year-Old Swedish Girls. Sex. Transm. Infect 2000; 76(2):98-102.

7. GIR, E.; MORIYA, T.M.; HAYASHIDA, M.; DUARTE, G.; MACHADO, A.A. Medidas Preventivas contra a AIDS e outras Doenças Sexualmente Transmissiveis Conhecidas por Univesitários da Área de Saúde. Ver. Lat. Am. Enfermagem 1999; 7(1):11-7.

8. HYDE, J.S. Understanding Human Sexuality. $5^{\mathrm{a}}$ ed. New York: McGraw-Hill Inc., 1992.

9. HARDY, E.E. Características Atuais Associadas à História de Aborto Provocado. Ver. Saúde pública 1994; 28(1):82-5.

10. MACHADO, A.A.; GIR, E.; DUARTE, G.; ANDREGHETTO, A.C.; CUNHA, A.A.; MIGUEL, C.E. et al. Avaliação do conhecimento sobre doenças sexualmente transmissiveis (DST) e síndrome da imunodeficiência adquirida (AIDS) entre Universitários de Ribeirão Preto - São Paulo. DST J. Bras. Doenças Sex. Transm. 1997, 9(6):12-6.

11. MAGALHÃES, M.L.C.; ANDRADE, H.H.S.M. Ginecologia Infanto Juvenil. Rio de Janeiro: Ed. Medsi, 1998.

12. MARTINEZ, C.; BENGIÓ, R.; DIAZ, M.; ARANCIBIA, M.; ALVES DA COSTA, P.; FERNÁNDEZ, R. La Sexualidad en Estudiantes Universitarios: Investigación de Comportamientos. Ver. Chil. Urol 1995, 60(2):201-4.

13. MASTERS, W.H.; JONHSON, V.; KOLODNY, R.C. Human Sexuality. New York: Harpers Collins Publishers, 1995.

14. PIATO, S. Ginecologia da Infância e Adolescência. São Paulo: Livraria Atheneu Editora, 2001.

15. POPPEN, P.J.; REISEN, C.A. Women's use of dual methods of sexual selfprotection. Women Health 1999; 30(2):53-66.

16. SADIGURSKY, C.A.; PEREIRA, F.C.; LOPES, J.R.P. O Inconsistente Uso do Preservativo na Adolescência. Publicado nos Anais do V Congresso Brasileiro da Sociedade de Obstetrícia e Ginecologia da Infância e da Adolescência, Porto Alegre, RS, 2000.

17. SADIGURSKY, D.; SADIGURSKY, C.A. Sexualidade na Adolescência: Um Estudo Realizado na População Atendida no CAISA. Publicado nos Anais do II Congresso Baiano da Sociedade de Reprodução Humana, 1998. 
18. SADIGURSKY, C.A.; COSTA, J.S. Gravidez na Adolescência: Integração Pais e filhos como Estratégia de Prevenção. Publicado nos Anais do V Congresso Brasileiro da Sociedade de Obstetrícia e Ginecologia da Infância e da Adolescência, Fortaleza, Ceará, 1998.

19. SAMPAIO, L.F. Sexualidade em Alunos de Medicina. Publicado nos Anais do VII Congresso Brasileiro de Sexualidade Humana, Rio de Janeiro, maio de 1999.

20. SOUZA, R.P.; ALMEIDA, A.B.; WAGNER, M.B.; ZIMERMAN, I.I.; ALMEIDA, S.B.; GONÇALVES, F.K. et al. Estudo sobre o comportamento sexual do jovem universitário de Porto Alegre (UFRGS/PUC/RS). Ver. AMRIGS 1987; 31(3):203-7.

21. VEJA FRANCO, E.E.; COSTA, R.G.; RODRIGUES, T.; MORAES, T.M. The religion concepts on induced aborting in young women. Ver. Mex. Pediatr 1997; 64(2):68-74.

22. VITIELLO, N. Sexologia II - Comissão Nacional da Febrasgo. São Paulo: CEICH, 1995.

23. VITIELLO, N.; CONCEIÇÃO, I.S.C.; CANELLA, P.R.B.; CAVALCANTI, R.C. Gestação na Adolescência. In: Adolescência Hoje, Comissão de Estudos sobre Adolescência. São Paulo: Roca, 1994.

24. VITIELLO, N. O Exercício da Sexualidade na Adolescência. In: Reprodução e Sexualidade: Manual para Educadores. São Paulo: CEICH 1994. 


\section{ANEXO}

Tabela 1 - Idade da coitarca entre 210 estudantes da Universidade Federal da Bahia, pacientes do Serviço Médico Universitário Rubens Brasil, 1995-1999.

\begin{tabular}{|l|r|c|}
\hline Idade & Número & Percentual \\
\hline 14 anos & 1 & 0,5 \\
15 anos & 8 & 3,8 \\
16 anos & 20 & 9,5 \\
17 anos & 43 & 20,5 \\
18 anos & 50 & 23,8 \\
19 anos & 28 & 13,3 \\
20 anos & 34 & 16,2 \\
ignorado & 26 & 12,4 \\
Total & $\mathbf{2 1 0}$ & $\mathbf{1 0 0}$ \\
\hline
\end{tabular}

Tabela 2 - Métodos contraceptivos usado por 172 estudantes da Universidade Federal da Bahia, pacientes do Serviço Médico Universitário Rubens Brasil, 1995-1999.

\begin{tabular}{|l|c|c|}
\hline Método & Número & Percentual \\
\hline Preservativo & 101 & 58,7 \\
Hormonal oral & 57 & 33,2 \\
Hormonal injetável & 10 & 5,8 \\
Ogino-Knaus & 4 & 2,3 \\
Total & $\mathbf{1 7 2}$ & $\mathbf{1 0 0}$ \\
\hline
\end{tabular}

